\title{
Stato di salute degli italiani e prospettive di spesa sanitaria e farmaceutica
}

Mario Eandi*

\section{PROSPETTIVE}

\begin{abstract}
In december 2000 the Italian National Institute of Statistics (INSTAT) published some extracts from the latest statistical survey about the state of health and the resort to medical services in Italy. The research is based on a sample of 52.300 italian families. Since 1980 the average lifetime in Italy grew five years longer: today the average expectancy for a man is 76 years old, and for a woman is 82 years old. All these deep demographic transformations involve an important increase in the chronic-degenerative pathologies. This rise will take up a great deal of public and private health resources.

The most frequent chronic-degenerative pathologies are arthrosis or arthritis (18\% of italian people) and the high blood pressure $(11,5 \%)$. After the age of 45 , the chronic-degenerative pathologies show a clear growth: some diseases are prevalent in women (e.g. osteoporosis), other prevail in men (e.g. chronic brochitis and stomach ulcer).

To better understand the intensity of the resort to medical services, the statistical survey also consider the perception of the health state in Italy.

The overall analysis show that the drug consumption (and the health resources consumption generally) is based on a complexity of social and cultural elements; therefore in some particular areas the control of the medical expenditure is more diffucult then in others.
\end{abstract}

Farmeconomia e percorsi terapeutici 2000; 1 (2): $71-81$

A metà dicembre 2000 l'Istat, Istituto Nazionale di Statistica, ha reso pubblica un'anticipazione dei risultati dell'ultima indagine Multiscopo condotta nel 1999/2000 su un campione di 52.300 famiglie italiane per studiare le "Condizioni di salute e ricorso ai servizi sanitari" della popolazione italiana.

Negli ultimi decenni la popolazione italiana è rapidamente invecchiata, con un allungamento della vita media di oltre 5 anni dal 1980 oggi gli uomini italiani hanno una prospettiva di vita di 76 anni e le donne di 82. Le profonde trasformazioni demografiche in atto (tra pochi anni si prevede che il $20 \%$ della popolazione italiana avrà un' età > 65 anni) comportano un aumento di patologie cronico-degenerative che assorbiranno una quantità crescente di risorse sanitarie pubbliche e private.

La tabella 1 riporta la prevalenza delle patologie cronico-degenerative più frequenti denunciate dal campione di italiani intervistati dall'Istat nel settembre-dicembre 1999. La quota di popolazione che dichiara di soffrire di almeno una malattia cronica supera il $47 \%$ e risulta lievemente maggiore fra le donne $(50,9 \%)$ rispetto agli uomini (43\%). Le artrosi e le artriti sono denunciate da oltre il $18 \%$ degli italiani. Al secondo posto troviamo l'ipertensione, de- nunciata dall' $11,5 \%$ della popolazione. Seguono, in ordine decrescente, le malattie allergiche, le cefalee, le lombosciatalgie, le vene varicose e le emorroidi, quindi l'osteoporosi, le broncopneumopatie croniche, la depressione e altri disturbi psichici, il diabete e via via molte altre patologie che, una volta insorte, caratterizzano in modo permanente la salute e la qualità di vita di un soggetto.

La maggior parte delle patologie croniche manifestano un netto incremento della diffusione con l'età, a partire dai 45 anni: fanno eccezione le malattie allergiche, l' asma bronchiale, le malattie della pelle e in parte la bronchite cronica, che colpiscono in numero elevato o prevalente anche soggetti giovani.

Alcune patologie, come l'osteoporosi, sono nettamente prevalenti nel sesso femminile, altre, come la bronchite cronica e l'ulcera gastrica, sono nettamente prevalenti nel sesso maschile. La ipertrofia prostatica benigna colpisce il 3,4\% della popolazione maschile.

L'indagine Istat ha stimato che siano circa 2.700.000 le persone disabili in Italia, pari al 4,8\% della popolazione con età maggiore ai 5 anni (Tabella 2): il sesso femminile è svantaggiato perché presenta un tasso di disabilità $(6,5 \%)$ nettamente superiore a quello degli uomini
* Dipartimento di Anatomia, Farmacologia e Medicina Legale, Sezione di Farmacologia, Università di Torino 
$(3,7 \%)$. La disabilità è stata definita in base alla scala ADL dell'OMS come presenza di una grave difficoltà in almeno una delle seguenti dimensioni: sconfinamento, difficoltà di movimento, difficoltà nelle funzioni quotidiane, difficoltà nella comunicazione. Il numero di disabili così stimato non comprende i soggetti con età minore ai sei anni e i circa 400.000 individui che presentano seri problemi di incontinenza non associati ad altre forme di disabilità. Anche la disabilità evidenzia una netta tendenza all'incremento con l'avanzare dell' età.

La disabilità è fortemente associata a forme patologiche di tipo cronico-degenerativo: 1'87\% delle persone disabili risulta essere affetto da almeno una patologia cronica, contro il $47 \%$ delle persone di sei anni o più che non presenta disabilità.
L'indagine Istat ha cercato di verificare anche quale sia la percezione dello stato di salute da parte della popolazione italiana. Infatti la salute è il risultato di una complessa interazione tra presenza-assenza di malattie o infermità e lo stato di benessere psichico e relazionale dell'individuo. L'autovalutazione dello stato di salute è importante non solo per comprendere le modificazioni della qualità di vita correlate a una patologia, ma anche per comprendere l'intensità del ricorso ai servizi sanitari.

Alla domanda "come va in generale la sua salute" il 60,8\% degli italiani ha risposto "bene" o "molto bene", e solo il 7,1\% ha risposto "male" o "molto male" (Tabella 3): il sesso femminile tende ad avere una percezione peggiore del proprio stato di salute rispetto al sesso maschile.

Per entrambi i sessi la percezione negativa
Tabella 1

Prevalenza perfasce di età e su intera popolazione delle patologie croniche più frequenti dichiarate dal campione di italiani intervistati nel periodo settembre/dicembre 1999

* Solo su popolazione maschile

(Fonte: Indagine Multiscopo ISTAT 1999-2000 su "Condizioni di salute e ricorso ai servizi sanitari”).

\begin{tabular}{|c|c|c|c|c|c|c|c|c|c|c|}
\hline \multirow{2}{*}{ Patologie } & \multicolumn{9}{|c|}{ Prevalenza per classi di età Campione } & \multirow{2}{*}{$\begin{array}{c}\text { Campione } \\
\text { Totale }\end{array}$} \\
\hline & $<14$ & $14-24$ & $25-34$ & $35-44$ & $45-54$ & $55-64$ & $65-74$ & $75-79$ & 380 & \\
\hline Artrosi, artrite & 0,0 & 0,6 & 3,4 & 8,9 & 22,1 & 36,2 & 49,0 & 54,5 & 58,9 & 18,3 \\
\hline Ipertensione & 0,0 & 0,4 & 1,6 & 3,8 & 11,9 & 24,4 & 33,3 & 38,4 & 36,6 & 11,5 \\
\hline $\begin{array}{l}\text { Malattie } \\
\text { allergiche }\end{array}$ & 8,3 & 11,3 & 12,3 & 11,2 & 9,2 & 8,1 & 8,1 & 7,5 & 5,3 & 9,7 \\
\hline Cefalee & 0,8 & 4,9 & 9,5 & 12,2 & 13,3 & 12,4 & 11,0 & 11,6 & 11,2 & 9,3 \\
\hline Lombosciatalgia & 0,1 & 0,8 & 4,6 & 8,0 & 12,4 & 14,8 & 15,1 & 16,8 & 15,5 & 8,2 \\
\hline Vene varicose & 0,0 & 0,7 & 2,6 & 5,1 & 8,4 & 13,0 & 15,3 & 16,7 & 16,6 & $\overline{66,7}$ \\
\hline Emorroidi & 0,1 & 1,4 & 4,7 & 6,1 & 8,3 & 9,3 & 8,7 & 9,5 & 9,3 & 5,6 \\
\hline Osteoporosi & 0,0 & 0,0 & 0,3 & 0,5 & 3,1 & 9,4 & 14,2 & 18,7 & 23,3 & 4,6 \\
\hline $\begin{array}{l}\text { Broncopatie } \\
\text { croniche }\end{array}$ & 0,6 & 0,8 & 1,0 & 1,7 & 3,6 & 7,5 & 12,6 & 15,0 & 19,0 & 4,5 \\
\hline $\begin{array}{c}\text { Depressione, } \\
\text { ecc. }\end{array}$ & 0,1 & 1,7 & 2,2 & 3,0 & 5,2 & 5,4 & 7,2 & 9,1 & 9,3 & 3,8 \\
\hline Diabete & 0,1 & 0,2 & $\overline{0,4}$ & 0,8 & 2,9 & 7,5 & 111,7 & 12,6 & 12,6 & 3,6 \\
\hline Altre cardiopatie & 0,3 & 0,3 & 0,8 & 0,9 & 1,8 & 5,1 & 9,9 & 14,7 & 17,7 & 3,4 \\
\hline $\begin{array}{c}\text { Malattie della } \\
\text { pelle }\end{array}$ & 1,4 & 2,6 & 3,3 & 3,5 & 3,8 & 3,8 & 4,0 & 3,4 & 5,0 & 3,3 \\
\hline Cataratta & 0,1 & 0,1 & 0,2 & 0,2 & 0,8 & 2,5 & 10,1 & 18,8 & 28,3 & 3,2 \\
\hline Asma bronchiale & 2,1 & 1,9 & $1,1,9$ & 1,7 & 2,0 & 3,8 & 5,8 & 7,7 & 8,4 & 3,0 \\
\hline Ulcera peptica & 0,0 & 0,2 & 1,1 & $1,1,9$ & 4,2 & 25,7 & 6,9 & 8,2 & 7,8 & 3,0 \\
\hline $\begin{array}{l}\text { Malattie della } \\
\text { tiroide }\end{array}$ & 0,1 & 0,6 & 1,3 & 2,8 & 4,4 & 5,2 & 5,0 & 4,5 & 3,6 & 2,7 \\
\hline Calcolosi biliare & 0,0 & 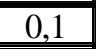 & 0,3 & 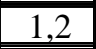 & 32,6 & $\begin{array}{l}4,4 \\
\end{array}$ & 6,6 & (6,4 & 6,6 & 2,3 \\
\hline Ernia addominale & 0,1 & 0,1 & 0,5 & 0,8 & 2,0 & 3,3 & 4,8 & 6,9 & 8,9 & 1,9 \\
\hline Calcolosi renale & 0,0 & 0,2 & 0,8 & 1,2 & 2,1 & 2,6 & 3,2 & 3,1 & 2,6 & 1,4 \\
\hline $\begin{array}{l}\text { Infarto del } \\
\text { miocardio }\end{array}$ & 0,0 & 0,0 & 0,0 & 0,2 & 1,1 & 2,5 & 3,7 & 4,9 & 4,3 & 1,2 \\
\hline $\begin{array}{l}\text { Parkinson, } \\
\text { Alzheimer }\end{array}$ & 0,1 & 0,2 & 0,4 & 0,3 & 0,6 & 0,8 & 2,3 & 4,3 & 10,6 & 1,1 \\
\hline Angina pectoris & 0,0 & 0,0 & 0,0 & 0,1 & 0,5 & $1,1,8$ & 3,0 & 4,4 & $4,4,3$ & 0,9 \\
\hline Tumore maligno & 0,0 & 0,0 & 0,1 & 0,3 & 1,0 & 1,9 & 2,6 & 2,8 & 2,7 & 0,9 \\
\hline $\begin{array}{c}\text { Trombosi, } \\
\text { embolia }\end{array}$ & 0,0 & 0,0 & 0,1 & 0,2 & 0,6 & 1,1 & 2,4 & 3,6 & 5,7 & 0,8 \\
\hline Cirrosi epatica & 0,0 & 0,0 & 0,0 & 0,1 & 0,2 & 0,5 & 0,9 & 0,9 & 0,3 & 0,2 \\
\hline Epatite cronica & 0,1 & 0,1 & 0,2 & 0,5 & 0,7 & 1,7 & 1,8 & $1,1,8$ & 1,1 & 0,7 \\
\hline $\begin{array}{c}\text { Ipertrofia della } \\
\text { prostata }\end{array}$ & 0,0 & 0,0 & 0,1 & 0,3 & 1,5 & 6,2 & 14,1 & 19,8 & 21,2 & $3,4^{*}$ \\
\hline
\end{tabular}

* Solo su popolazione maschile 


\begin{tabular}{|c|c|c|c|c|c|c|c|c|c|}
\hline & \multicolumn{8}{|c|}{ Prevalenza di disabili per classi di età (anni) } & \multirow{2}{*}{ CampioneTotale } \\
\hline & $6-14$ & $15-24$ & $25-44$ & $45-54$ & $55-64$ & $65-74$ & $75-79$ & 380 & \\
\hline MASCHI & 1,50 & 0,84 & 1,09 & 1,74 & 3,47 & 7,33 & 16,01 & 38,50 & 3,55 \\
\hline FEMMINE & 1,48 & 1,03 & 0,99 & 1,53 & 4,58 & 10,91 & 23,91 & 51,67 & 6,33 \\
\hline TOTALE & 1,49 & 0,94 & 1,04 & 1,64 & 4,04 & 9,31 & $\mid 20,72$ & 47,50 & 4,98 \\
\hline
\end{tabular}

del proprio stato di salute tende ad aumentare con l'età.

L'area geografica di residenza sembra in parte influenzare la percezione del proprio stato di salute (Tabella 3). Nelle cinque diverse ripartizioni geografiche dell'Italia la quota di popolazione che dichiara di stare "discretamente" è piuttosto stabile e oscilla attorno al $30 \%$; al contrario, distanze rilevanti si riscontrano per le modalità estreme. In particolare, nell'Italia Meridionale e Insulare si trova una quota maggiore di persone che dichiarano di stare "male" o "molto male", mentre nell'Italia Settentrionale un maggior numero di persone dichiara di stare "bene" o "molto bene".

La percentuale di anziani che valuta negativamente il proprio stato di salute tende a diminuire risalendo la penisola fino ai valori minimi del Nord-Ovest. Le condizioni soggettive dello stato di salute sono fortemente correlate al titolo di studio: il fatto che la popolazione anziana residente al Sud presenti mediamente livelli di istruzione più bassi rispetto ai residenti nelle altre ripartizioni spiega almeno in parte lo svantaggio evidenziato.

La valutazione soggettiva del proprio stato di salute è decisamente influenzata dalla presenza di malattie croniche e dalla presenza di disabilità. La Figura 1 evidenzia la relazione tra frequenza di risposte sulla percezione dello stato di salute e assenza o presenza di uno o più malattie croniche. La percezione negativa delle proprie condizioni di salute cresce con l'aumentare del numero di malattie croniche presenti; un comportamento specularmente opposto riguarda invece la percezione positiva della salute.

La presenza di disabilità incide sensibilmente sulla percezione dello stato di salute al crescere dell'età (Tabella 4). Nella popolazione non disabile con l'aumentare dell'età diventa maggiore la quota di persone che si colloca nella modalità "discretamente": questa modalità diventa prevalente nei soggetti più anziani. Nella popolazione disabile, invece, già nei giovani si hanno quote elevate di soggetti che percepiscono negativamente il proprio stato di salute e tale quota aumenta con l'età: oltre i 55 anni la modalità "male" o "molto male" rappresenta la condizione prevalente tra i disabili.

I risultati dell'indagine Istat confermano come la domanda di assistenza sanitaria da parte della popolazione italiana è destinata ad aumentare nei prossimi anni, ponendo in forte tensione il sistema di finanziamento pubblico della Sanità e dell'intero Sistema di Protezione Sociale.

Attualmente il Sistema di Protezione Socia-
Tabella 2

Distribuzione della prevalenza di disabili per classi di età e sesso in un campione rappresentativo della popolazione italiana (Fonte: Indagine Multiscopo ISTAT 1999-2000 su "Condizioni di salute $e$ ricorso ai servizi sanitari").

\begin{tabular}{|c|c|c|c|c|}
\hline & & $\begin{array}{c}\text { Male o } \\
\text { molto male }\end{array}$ & Discretamente & $\begin{array}{c}\text { Bene o } \\
\text { molto bene }\end{array}$ \\
\hline \multicolumn{2}{|l|}{ TOTALE ITALIA } & $7,1 \%$ & $32,0 \%$ & $60,9 \%$ \\
\hline \multicolumn{2}{|l|}{ Maschi } & $5,6 \%$ & $28,7 \%$ & $65,7 \%$ \\
\hline \multicolumn{2}{|l|}{ Femmine } & $8,5 \%$ & $35,1 \%$ & $56,4 \%$ \\
\hline \multicolumn{5}{|l|}{ RIPARTIZIONI } \\
\hline \multirow[t]{2}{*}{ Italia Nord-Occidentale } & (tasso grezzo) & $6,10 \%$ & $5,82 \%$ & $33,60 \%$ \\
\hline & (tasso standardizzato) & $31,39 \%$ & $60,30 \%$ & $61,14 \%$ \\
\hline \multirow[t]{2}{*}{ Italia Nord-Orientale } & (tasso grezzo) & $6,50 \%$ & $4,86 \%$ & $34,50 \%$ \\
\hline & (tasso standardizzato) & $29,49 \%$ & $59,00 \%$ & $63,56 \%$ \\
\hline \multirow[t]{2}{*}{ Italia Centrale } & (tasso grezzo) & $8,10 \%$ & $7,43 \%$ & $33,60 \%$ \\
\hline & (tasso standardizzato) & $32,57 \%$ & $58,30 \%$ & $50,02 \%$ \\
\hline \multirow[t]{2}{*}{ Italia Meridionale } & (tasso grezzo) & $7,00 \%$ & $8,14 \%$ & $27,90 \%$ \\
\hline & (tasso standardizzato) & $31,89 \%$ & $65,20 \%$ & $58,87 \%$ \\
\hline \multirow[t]{2}{*}{ Italia Insulare } & (tasso grezzo) & $9,00 \%$ & $10,13 \%$ & $30,30 \%$ \\
\hline & (tasso standardizzato) & $31,67 \%$ & $60,70 \%$ & $56,24 \%$ \\
\hline
\end{tabular}

Tabella 3

Distribuzione della popolazione italiana secondo lo stato di salute dichiarato come percezione soggettiva. (Fonte: Indagine Multiscopo ISTAT 1999-2000 su "Condizioni di salute e ricorso ai servizi sanitari"). 


\section{Figura 1}

Relazione tra numero dichiarato di malattie croniche presenti e percezione soggettiva dello stato di salute in un campione rappresentativo della popolazione italiana (Fonte: Indagine Multiscopo ISTAT 1999-2000 su "Condizioni di salute $e$ ricorso ai servizi sanitari").

Tabella 4

Distribuzione percentuale della percezione soggettiva dello stato di salute in funzione dell'età e della presenza o assenza di disabilità in un campione rappresentativo della popolazione italiana (Fonte: Indagine Multiscopo ISTAT 1999-2000 su "Condizioni di salute e ricorso ai servizi sanitari”).

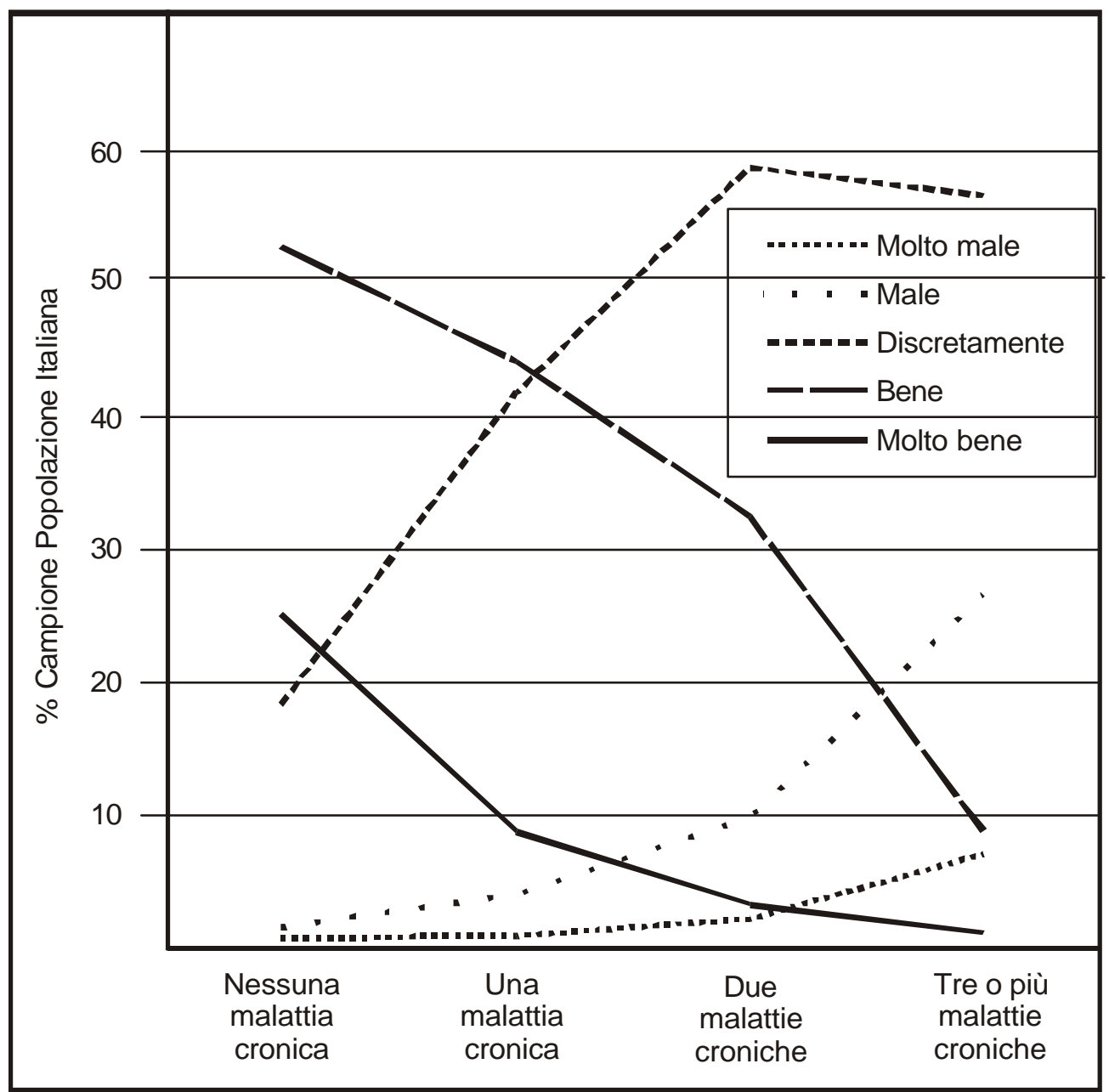

\begin{tabular}{|c|c|c|c|c|}
\hline \multirow[b]{2}{*}{ Classe di età } & \multirow[b]{2}{*}{ Disabilità } & \multicolumn{3}{|c|}{ Stato di Salute Percepito } \\
\hline & & $\begin{array}{c}\text { Male o } \\
\text { molto male }\end{array}$ & Discretamente & $\begin{array}{c}\text { Bene o } \\
\text { molto bene }\end{array}$ \\
\hline \multirow{2}{*}{$6-14$} & Presente & $3,3 \%$ & $0,6 \%$ & $14,4 \%$ \\
\hline & Assente & $6,1 \%$ & $82,3 \%$ & $93,3 \%$ \\
\hline \multirow{2}{*}{$15-24$} & Presente & $26,9 \%$ & $1,1 \%$ & $22,3 \%$ \\
\hline & Assente & $12,9 \%$ & $50,8 \%$ & $86,0 \%$ \\
\hline \multirow{2}{*}{$25-44$} & Presente & $33,6 \%$ & $1,8 \%$ & $35,4 \%$ \\
\hline & Assente & $24,3 \%$ & $31,0 \%$ & $73,9 \%$ \\
\hline \multirow{2}{*}{$45-54$} & Presente & $40,2 \%$ & $5,2 \%$ & $42,4 \%$ \\
\hline & Assente & $42,4 \%$ & $17,3 \%$ & $52,4 \%$ \\
\hline \multirow{2}{*}{$55-64$} & Presente & $53,3 \%$ & $8,9 \%$ & $40,2 \%$ \\
\hline & Assente & $52,8 \%$ & $6,5 \%$ & \\
\hline \multirow{2}{*}{$65-74$} & Presente & $64,3 \%$ & $13,4 \%$ & $31,9 \%$ \\
\hline & Assente & $62,0 \%$ & $3,8 \%$ & $24,5 \%$ \\
\hline \multirow{2}{*}{$>75$} & Presente & $61,0 \%$ & $15,2 \%$ & $35,0 \%$ \\
\hline & Assente & $65,3 \%$ & $4,0 \%$ & $19,5 \%$ \\
\hline \multirow{2}{*}{ Totale } & Presente & $55,6 \%$ & $4,9 \%$ & $34,4 \%$ \\
\hline & Assente & $33,3 \%$ & $10,0 \%$ & $61,8 \%$ \\
\hline
\end{tabular}




\begin{tabular}{|c|c|c|c|c|c|c|}
\hline \multirow{2}{*}{ Anno } & \multirow{2}{*}{$\begin{array}{c}\text { Prodotto } \\
\text { Interno } \\
\text { Lordo (PIL) } \\
\text { (miliardi L.) }\end{array}$} & \multirow{2}{*}{$\begin{array}{c}\text { Debito } \\
\text { Pubblico } \\
\text { Totale } \\
\text { (miliardi L.) }\end{array}$} & \multicolumn{4}{|c|}{$\begin{array}{c}\text { Spesa Pubblica per Protezione Sociale } \\
\text { (Welfare State) }\end{array}$} \\
\hline & & & (miliardi L.) & Sanità \% & Previdenza \% & Assistenza \% \\
\hline 1989 & 1.193 .462 & 1.219 .519 & 248.240 & $25,5 \%$ & $58,2 \%$ & $16,3 \%$ \\
\hline 1990 & 1.312 .066 & 1.384 .778 & 284.718 & $26,3 \%$ & $57,6 \%$ & $16,1 \%$ \\
\hline 1991 & 1.429 .453 & 1.562 .164 & 332.465 & $25,0 \%$ & $66,8 \%$ & $8,2 \%$ \\
\hline 1992 & 1.502 .493 & 1.752 .926 & 367.573 & $23,6 \%$ & $68,5 \%$ & $7,9 \%$ \\
\hline 1993 & 1.550 .296 & 1.927 .891 & 382.520 & $22,7 \%$ & $68,9 \%$ & $8,4 \%$ \\
\hline 1994 & 1.638 .666 & 2.105 .458 & 400.857 & $21,6 \%$ & $70,0 \%$ & $8,4 \%$ \\
\hline 1995 & 1.771 .018 & 2.258 .700 & 416.667 & $20,6 \%$ & $71,2 \%$ & $8,2 \%$ \\
\hline 1996 & 1.873 .494 & 2.319 .395 & 444.979 & $20,3 \%$ & $71,8 \%$ & $7,9 \%$ \\
\hline 1997 & 1.974 .618 & 2.372 .200 & 451.880 & $24,0 \%$ & $63,0 \%$ & $13,0 \%$ \\
\hline 1998 & 2.057 .731 & 2.413 .100 & 461.990 & $24,3 \%$ & $63,3 \%$ & $12,4 \%$ \\
\hline 1999* & 2.124 .476 & 2.458 .019 & 482.000 & $23,5 \%$ & $63,5 \%$ & $13,0 \%$ \\
\hline $2000^{*}$ & 2.206.013 & 2.490 .589 & 500.000 & $23,4 \%$ & $63,9 \%$ & $13,0 \%$ \\
\hline $2001 *$ & 2.298 .354 & 2.507 .504 & 515.600 & $23,4 \%$ & $63,9 \%$ & $12,7 \%$ \\
\hline $2002 *$ & 2.401 .400 & 2.511 .864 & 532.200 & $23,3 \%$ & $64,3 \%$ & $12,4 \%$ \\
\hline
\end{tabular}

Tabella 5

Evoluzione del Prodotto Interno Lordo, del debito Pubblico e della Spesa Pubblica per il Sistema di Protezione Sociale in Italia negli anni 1989-2003 (Fonte: Ministero del Tesoro, Documento di Programmazione Economica e Finanziaria 19992001 e 2000-2003).

\footnotetext{
* Quadro programmatico previsto dai D.P.E.F. 1999-2001 e 2000-2003.
}

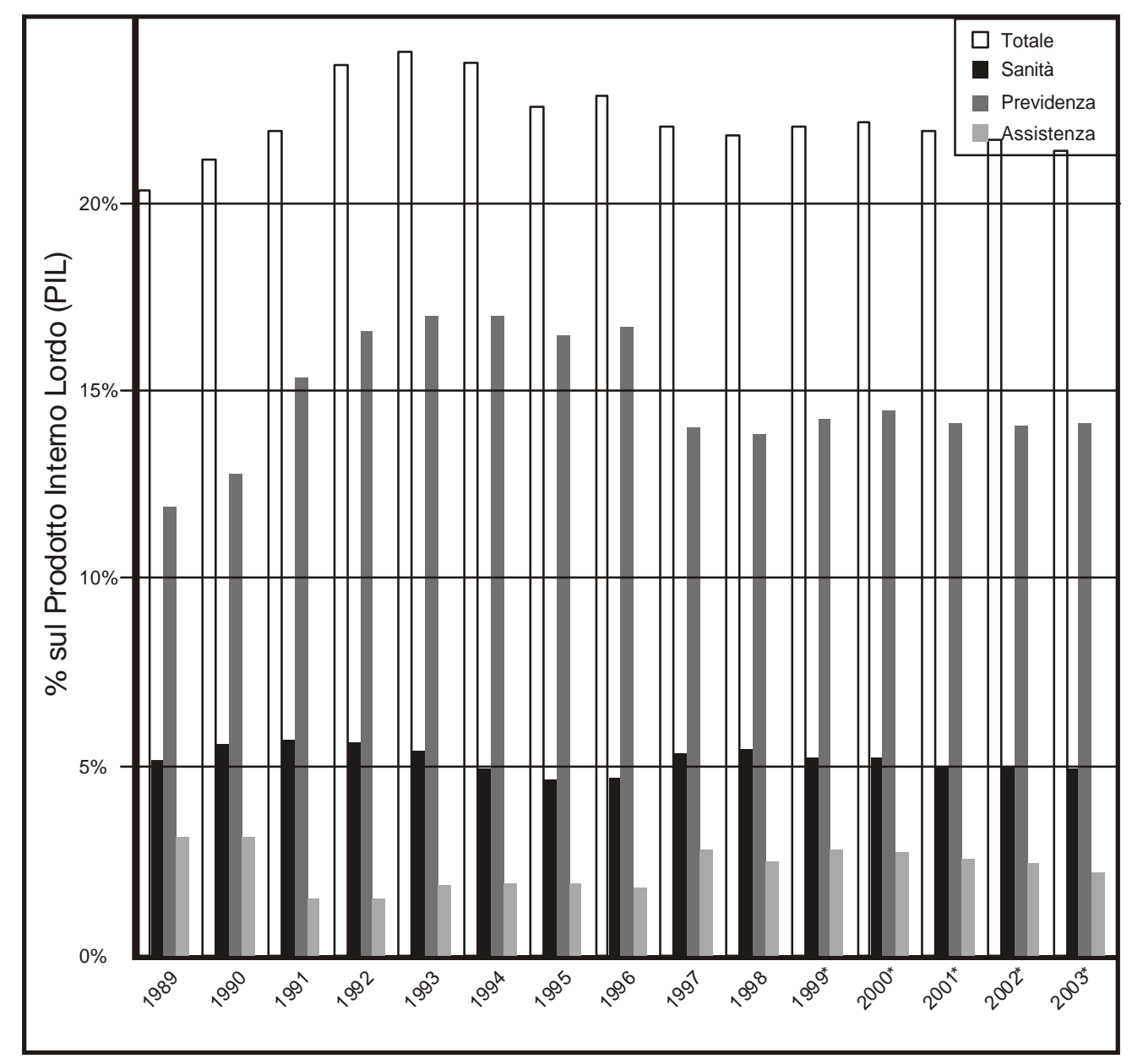

Figura 2

Evoluzione della Spesa Pubblica per il Sistema di Protezione Sociale in Italia, espresso come percentuale del PIL. I dati relativi agli anni 1999-2003 sono previsioni contenute nei D.P.E.F. 1999-2001 e 20002003. (Fonte: Ministero del Tesoro, Documento di Programmazione Economica e Finanziaria 1999-2001 e 2000-2003). 


\section{Tabella 6}

Spesa Sanitaria Totale Italiana e sua ripartizione tra pubblico e privato negli anni 1989-2003 (Fonte: Ministero del Tesoro, Documento di Programmazione Economica e Finanziaria 1999. 2001 e 2000-2003).

\section{Tabella 7}

Spesa Farmaceutica Convenzionata a carico del SSN italiano negli anni 1997-1999 (Fonte: Ministero della Sanità e Agenzia per $i$ Servizi Sanitari Regionali).

\begin{tabular}{|c|c|c|c|c|c|}
\hline \multirow{2}{*}{ Anno } & \multirow{2}{*}{$\begin{array}{c}\text { Spesa Sanitaria } \\
\text { Totale } \\
\text { (miliardi L.) }\end{array}$} & \multicolumn{2}{|c|}{ Spesa Sanitaria Pubblica } & \multicolumn{2}{|c|}{ Spesa Sanitaria Privata } \\
\hline & & (miliardi L.) & $\%$ & (miliardi L.) & $\%$ \\
\hline 1989 & 86.058 & 67.248 & $78,14 \%$ & 18.810 & $21,86 \%$ \\
\hline 1990 & 101.190 & 80.145 & $79,20 \%$ & 21.045 & $20,80 \%$ \\
\hline 1991 & 116.241 & 92.386 & $79,48 \%$ & 23.855 & $20,52 \%$ \\
\hline 1992 & 123.531 & 95.503 & $77,31 \%$ & 28.028 & $22,69 \%$ \\
\hline 1993 & 127.983 & 94.390 & $73,75 \%$ & 33.593 & $26,25 \%$ \\
\hline 1994 & 132.489 & 94.557 & $71,37 \%$ & 37.932 & $28,63 \%$ \\
\hline 1995 & 132.058 & 92.794 & $70,27 \%$ & 39.264 & $29,73 \%$ \\
\hline 1996 & 140.978 & 99.472 & $70,56 \%$ & 41.506 & $29,44 \%$ \\
\hline 1997 & 152.422 & 108.578 & $71,24 \%$ & 44.035 & $28,89 \%$ \\
\hline 1998 & 158.893 & 112.480 & $70,79 \%$ & 46.413 & $29,21 \%$ \\
\hline $1999 *$ & 163.308 & 116.150 & $71,12 \%$ & 47.158 & $28,88 \%$ \\
\hline $2000 *$ & 168.643 & 119.000 & $70,56 \%$ & 49.643 & $29,44 \%$ \\
\hline $2001 *$ & 172.816 & 120.688 & $69,84 \%$ & 52.128 & $30,16 \%$ \\
\hline $2002 *$ & 179.283 & 124.670 & $69,54 \%$ & 54.613 & $30,46 \%$ \\
\hline $2003 *$ & 185.883 & 128.785 & $69,28 \%$ & 57.098 & $30,72 \%$ \\
\hline
\end{tabular}

* Quadro programmatico previsto dai D.P.E.F. 1999-2001 e 2000-2003.

le (Welfare State) assorbe circa 500.000 miliardi stabile attorno al 5\% del PIL e pertanto l'incredi Lire, dei quali circa il $64 \%$ servono a finanzia- mento relativo rispetto all' incremento del PIL è re le pensioni e solo poco più del $23 \%$ a finan- tutto a carico dei privati. Come conseguenza la ziare l'assistenza sanitaria pubblica (tabella 5). compartecipazione del privato alla spesa saniL'Italia è appesantita da un enorme debito pub- taria in dieci anni è passata da circa il $20 \%$ a blico che solo nel 2003, se le previsioni degli oltre il 30\% (Tabella 6).

ultimi D.P.E.F verranno rispettate, andrà in pa- Com'è noto, il finanziamento pubblico delreggio con il Prodotto Interno Lordo (PIL). Con l'assistenza sanitaria italiana è inferiore di circa pesanti manovre finanziarie la spesa per lo Sta- 2-2,5 punti percentuali rispetto al finanziamento Sociale è stata posta sotto controllo negli to pubblico della maggior parte degli altri Paesi ultimi anni e oggi si è relativamente stabilizzata europei coi quali condividiamo il mercato e la appena oltre il $23 \%$ del PIL: tale opera di moneta unica Euro.

contenimento, tuttavia, è stata attuata anche e soprattutto mediante il taglio della spesa sanitaria pubblica e solo in parte mediante la riforma parziale del sistema pensionistico (vedi figura 2).

La spesa sanitaria totale italiana è in continuo aumento e oggi si aggira intorno ai 170.000 miliardi di Lire. La quota pubblica è mantenuta

La situazione demografica dell'Italia e lo stato di salute degli italiani richiedono tendenzialmente un finanziamento pubblico analogo a quello degli altri Paesi europei. Nei prossimi anni la quota pubblica della spesa sanitaria è destinata a entrare in forte tensione a causa delle spinte al rialzo introdotte dalle nuove tecnologie sempre molto costose e a cusa del livello

\begin{tabular}{|c|c|c|c|c|c|}
\hline & \multicolumn{3}{|c|}{ Spesa Farmaceutica Convenzionata (miliardi Lire) } & \multicolumn{2}{|c|}{ Incremento \% } \\
\hline & 1997 & 1998 & 1999 & 98 vs 97 & 99 vs 98 \\
\hline Spesa Netta & 12.106 & 13.203 & 14.691 & $9,06 \%$ & $11,27 \%$ \\
\hline Ticket & 1.460 & 1.570 & 1.585 & $7,53 \%$ & $0,96 \%$ \\
\hline Sconto Farmacie & 538 & 602 & 686 & $11,90 \%$ & $13,95 \%$ \\
\hline $\mathrm{N}^{\circ}$ Ricette & 296.000 .000 & 310.000 .000 & 327.000 .000 & $4,73 \%$ & $5,48 \%$ \\
\hline
\end{tabular}




\begin{tabular}{||l||r||r||r||r||c|}
\hline \multicolumn{1}{|c||}{ Classe } & $\begin{array}{r}\text { Spesa Lorda } \\
1997\end{array}$ & Incidenza \% & $\begin{array}{c}\text { Spesa Lorda } \\
1998\end{array}$ & Incidenza \% & $\begin{array}{c}\text { Incremento \% } \\
98 \text { vs 97 }\end{array}$ \\
\hline \hline A & 13.949 & $58,92 \%$ & 15.487 & $60,39 \%$ & $11,03 \%$ \\
B & 1.430 & $6,04 \%$ & 1.543 & $6,02 \%$ & $7,90 \%$ \\
\hline A+B & $\mathbf{1 5 . 3 7 9}$ & $\mathbf{6 4 , 9 6 \%}$ & $\mathbf{1 7 . 0 3 0}$ & $\mathbf{6 6 , 4 1 \%}$ & $\mathbf{1 0 , 7 4 \%}$ \\
\hline C: obbligo prescrizione & 4.975 & $21,02 \%$ & 5.269 & $20,55 \%$ & $5,91 \%$ \\
C: senza prescrizione & 1.397 & $5,90 \%$ & 1.371 & $5,35 \%$ & $-1,86 \%$ \\
C: prodotti da banco & 1.922 & $8,12 \%$ & 1.994 & $7,78 \%$ & $3,75 \%$ \\
\hline Totale C & $\mathbf{8 . 2 9 4}$ & $\mathbf{3 5 , 0 4 \%}$ & $\mathbf{8 . 6 3 4}$ & $\mathbf{3 3 , 6 7 \%}$ & $\mathbf{4 , 1 0 \%}$ \\
\hline \hline Totale Generale & $\mathbf{2 3 . 6 7 3}$ & $\mathbf{1 0 0 , 0 0 \%}$ & $\mathbf{2 5 . 6 4 4}$ & $\mathbf{1 0 0 , 0 0 \%}$ & $\mathbf{8 , 3 3 \%}$ \\
\hline
\end{tabular}

\section{Tabella 8}

Spesa Farmaceutica Lorda in Italia negli anni 1997-98, ripartita secondo le Classi A, B e C del Prontuario Terapeutico del SSN: dati di vendita in miliardi di Lire (Fonte: Ministero della Sanità). insufficiente delle risorse allocate al comparto sanitario dal bilancio dello Stato. In questo momento congiunturale della storia politica ed economica dell'Italia è difficile intravvedere soluzioni che non incidano direttamente o indirettamente sul portafoglio dei cittadini.

Il contenimento della spesa è molto difficile, sia per l'attuale situazione di grande sviluppo scientifico e tecnologico che crea espansione dell'offerta e quindi della domanda di assistenza, sia per la rigidità di alcuni comparti della sanità, come quello dell'assistenza ospedaliera, che da sola drena quasi il $60 \%$ dell'intera spesa sanitaria pubblica.

La spesa farmaceutica, che è stata contenuta drasticamente a partire dal 1993, denota da alcuni anni una forte tendenza all'espansione ben oltre i limiti programmati anno per anno

\section{dal Governo.}

A partire dal 1997 l'incremento annuale della spesa farmaceutica convenzionata netta e della spesa farmaceutica totale lorda è stato mediamente del 10\% (Tabelle 7 e 8). Il numero di ricette annuali è aumentato negli ultimi anni, mentre la compartecipazione sotto forma di ticket non ha seguito questo sviluppo e sembra essersi stabilizzata a poco meno di 1.600 miliardi di Lire.

Ultimamente circa il $65 \%$ della spesa farmaceutica lorda è dovuto a prescrizione di farmaci collocati in Classe A (a totale carico del SSN) e B (a carico del SSN per il 50\% del prezzo), mentre il $35 \%$ è dovuto a vendita dei prodotti in Classe $\mathrm{C}$, che sono a totale carico del paziente (Tabella 8). In termini di valore assoluto la quota di spesa farmaceutica dovuta alla prescrizio-

\begin{tabular}{|c|c|c|c|c|}
\hline & Gruppo Terapeutico & \begin{tabular}{|c} 
Spesa Lorda \\
1998
\end{tabular} & $\begin{array}{c}\% \\
(\text { su totale })\end{array}$ & $\begin{array}{c}\% \\
\text { Cumulativo }\end{array}$ \\
\hline 1 & $\begin{array}{l}\text { Antibatterici beta-lattamici: cefalosporine, } \\
\text { monobattami e carbapenemi }\end{array}$ & 1.159 & $6,82 \%$ & $6,82 \%$ \\
\hline 2 & Calcio-antagonisti selettivi sui vasi (diidropiridinici) & 1.076 & $6,33 \%$ & $13,14 \%$ \\
\hline 3 & Antiulcera peptica & 1.066 & $6,27 \%$ & $19,41 \%$ \\
\hline 4 & ACE-inibitori (non associati) & 1.027 & $6,04 \%$ & $25,44 \%$ \\
\hline 5 & FANS & 809 & $4,75 \%$ & $30,20 \%$ \\
\hline 6 & ACE-inibitori associati a diuretici & 797 & $4,69 \%$ & $34,88 \%$ \\
\hline 7 & Macrolidi e lincosamidi & 654 & $3,84 \%$ & $38,73 \%$ \\
\hline 8 & $\begin{array}{l}\text { Immunostimolanti (interferoni e fattori di } \\
\text { stimolazione delle colonie) }\end{array}$ & 559 & $3,28 \%$ & $42,01 \%$ \\
\hline 9 & Ipocolesterolemizzanti ed ipotrigligeridemizzanti & 515 & $3,03 \%$ & $45,04 \%$ \\
\hline 10 & Penicilline & 499 & $2,94 \%$ & $47,97 \%$ \\
\hline 11 & Vasodilatatori per cardiopatie (nitrati) & 428 & $2,52 \%$ & $50,49 \%$ \\
\hline 12 & $\begin{array}{l}\text { Antiasmatici per aerosol (glicocorticoidi, } \\
\text { anticolinergici, sostanze antiallergiche) }\end{array}$ & 417 & $2,45 \%$ & $52,94 \%$ \\
\hline 13 & Farmaci per l'ipertrofia prostatica benigna & 375 & $2,21 \%$ & $55,15 \%$ \\
\hline 14 & Adrenergici per aerosol & 356 & $2,09 \%$ & $57,24 \%$ \\
\hline 15 & Ormoni e sostanze correlate (reline, progestinici) & 345 & $2,03 \%$ & $59,27 \%$ \\
\hline & Totale primi 15 gruppi terapeutici & 10.083 & $59,27 \%$ & \\
\hline
\end{tabular}

\section{Tabella 9}

Primi 15 gruppi terapeutici in ordine decrescente di spesa farmaceutica lorda (quota a carico del SSN + quota per acquisto diretto) nel 1998: dati di vendita in miliardi di Lire (Fonte: Ministero della Sanità). 
Tabella 10

Primi 15 gruppi terapeutici in ordine di aumento assoluto della spesa farmaceutica lorda italiana (quota a carico del SSN + quota per acquisto diretto) nel 1998 in confronto con l'anno precedente: dati di vendita in miliardi di Lire (Fonte: Ministero della Sanità).

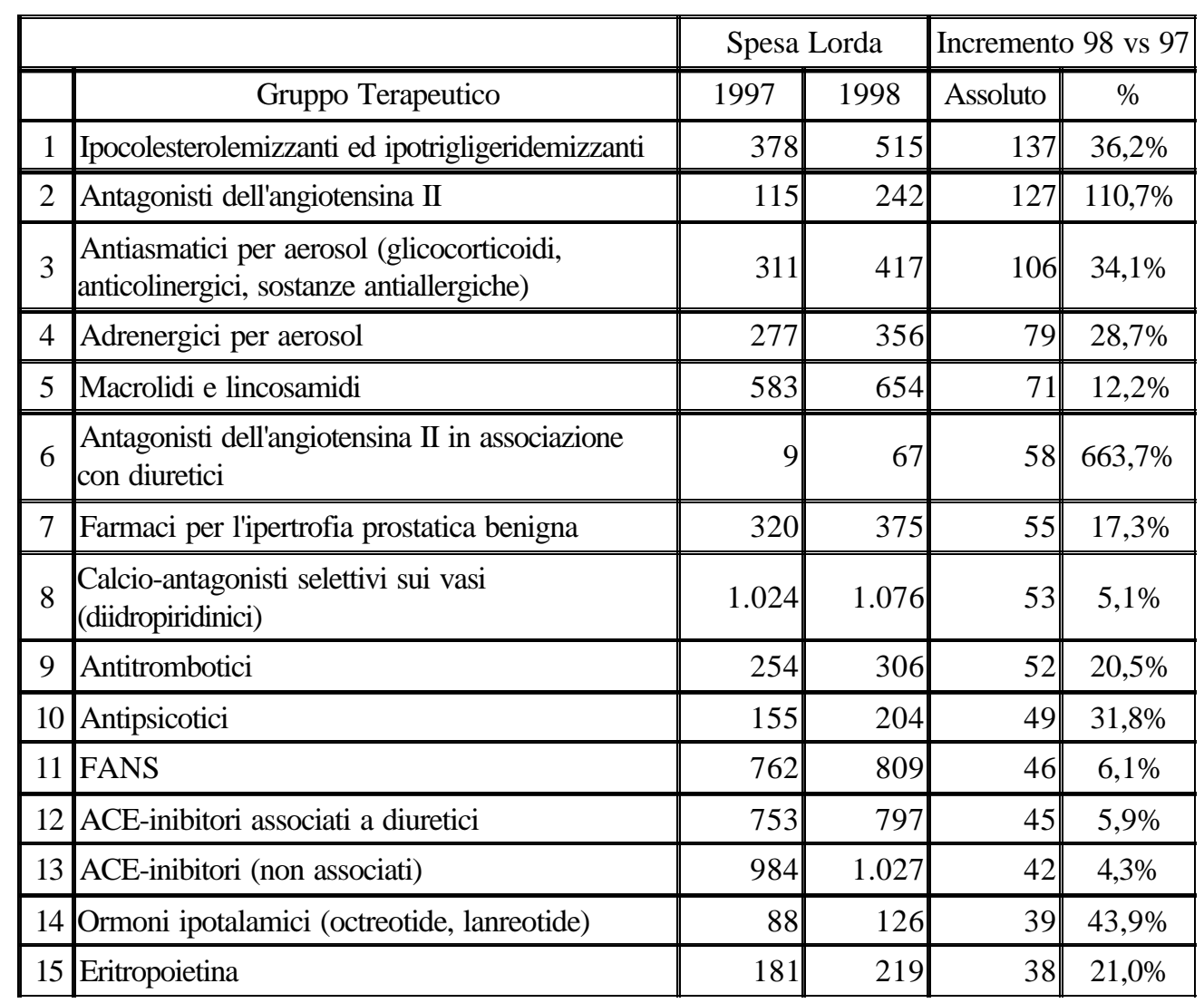

\section{Tabella 11}

Spesa farmaceutica convenzionata a carico del SSN italiano nel 1999 ed incrementi $\%$ rispetto al 1998: ripartizione per Regioni (Fonte: Agenzia per i Servizi Sanitari Regionali)

\begin{tabular}{|c|c|c|c|c|c|c|c|c|}
\hline & \multicolumn{2}{|c|}{ Spesa Netta 1999} & \multicolumn{2}{|c|}{ Ticket 1999} & \multicolumn{2}{|c|}{ Sc. Farmacie 1999} & \multicolumn{2}{|c|}{ N $^{\circ}$ Ricette 1999} \\
\hline & (milioni) & $\begin{array}{c}\text { Incr. \% } \\
\text { vs } 98\end{array}$ & (milioni) & $\begin{array}{c}\text { Incr. } \% \\
\text { vs } 98\end{array}$ & (milioni) & $\begin{array}{c}\text { Incr. \% } \\
\text { vs } 98\end{array}$ & (migliaia) & $\begin{array}{c}\text { Incr. \% } \\
\text { vs } 98\end{array}$ \\
\hline V. AOSTA & 26.853 & $16,0 \%$ & 2.955 & $9,6 \%$ & 838 & $2,7 \%$ & 586 & $6,2 \%$ \\
\hline PIEMONTE & 1.058 .790 & $13,9 \%$ & 115.224 & $9,4 \%$ & 47.518 & $3,9 \%$ & 22.504 & $7,0 \%$ \\
\hline LIGURIA & 478.899 & $9,8 \%$ & 50.497 & $9,1 \%$ & 23.124 & $4,2 \%$ & 10.240 & $5,5 \%$ \\
\hline LOMBARDIA & 2.091 .460 & $10,3 \%$ & 232.879 & $9,6 \%$ & 100.959 & $4,2 \%$ & 43.163 & $4,8 \%$ \\
\hline VENETO & 976.857 & $13,2 \%$ & 117.362 & $10,3 \%$ & 43.109 & $3,8 \%$ & 22.347 & $6,8 \%$ \\
\hline BOLZANO & 87.852 & $23,7 \%$ & 9.466 & $9,4 \%$ & 3.650 & $3,6 \%$ & 1.808 & $10,2 \%$ \\
\hline TRENTO & 89.924 & $9,4 \%$ & 9.818 & $9,5 \%$ & 3.506 & $3,4 \%$ & 1.963 & $3,8 \%$ \\
\hline FRIULI V.G. & 266.595 & $14,6 \%$ & 30.017 & $9,7 \%$ & 11.609 & $3,8 \%$ & 6.063 & $7,5 \%$ \\
\hline E. ROMAGNA & 926.394 & $14,6 \%$ & 115.605 & $10,7 \%$ & 39.714 & $3,7 \%$ & 23.129 & $6,8 \%$ \\
\hline MARCHE & 381.446 & $7,9 \%$ & 42.132 & $9,6 \%$ & 15.998 & $3,6 \%$ & 8.975 & $3,8 \%$ \\
\hline TOSCANA & 858.400 & $10,4 \%$ & 104.401 & $10,4 \%$ & 38.397 & $3,8 \%$ & 21.671 & $6,0 \%$ \\
\hline LAZIO & 1.496 .902 & $10,8 \%$ & 159.779 & $9,2 \%$ & 77.025 & $4,4 \%$ & 32.045 & $6,7 \%$ \\
\hline UMBRIA & 236.906 & $10,4 \%$ & 24.331 & $9,0 \%$ & 9.169 & $3,4 \%$ & 5.533 & $5,9 \%$ \\
\hline ABRUZZO & 354.893 & $15,9 \%$ & 35.943 & $8,9 \%$ & 14.045 & $3,5 \%$ & 8.027 & $8,3 \%$ \\
\hline MOLISE & 82.235 & $15,5 \%$ & 8.358 & $8,9 \%$ & 3.168 & $3,4 \%$ & 1.787 & $8,8 \%$ \\
\hline CAMPANIA & 1.668 .900 & $7,7 \%$ & 163.019 & $8,5 \%$ & 80.172 & $4,2 \%$ & 36.485 & $2,0 \%$ \\
\hline PUGLIA & 1.073 .952 & $10,0 \%$ & 102.692 & $8,3 \%$ & 55.560 & $4,5 \%$ & 23.340 & $6,2 \%$ \\
\hline BASILICATA & 152.819 & $11,0 \%$ & 15.348 & $8,8 \%$ & 5.948 & $3,4 \%$ & 3.504 & $6,3 \%$ \\
\hline CALABRIA & 545.616 & $8,5 \%$ & 52.124 & $8,4 \%$ & 21.714 & $3,5 \%$ & 12.730 & $9,2 \%$ \\
\hline SICILIA & 1.437 .087 & $13,4 \%$ & 149.145 & $9,0 \%$ & 72.235 & $4,4 \%$ & 32.637 & $7,4 \%$ \\
\hline SARDEGNA & 398.036 & $14,8 \%$ & 43.508 & $9,5 \%$ & 18.611 & $4,0 \%$ & 8.898 & $7,2 \%$ \\
\hline ITALIA & 14.690 .816 & $11,3 \%$ & 1.584 .603 & $9,3 \%$ & 686.069 & $4,0 \%$ & 327.435 & $6,0 \%$ \\
\hline
\end{tabular}


ne di farmaci di Classe A e B è aumentata maggiormente in termini percentuali rispetto alla quota dovuta alla vendita dei farmaci di Classe C. Nella Classe A sono collocati in genere tutti i farmaci innovativi, la maggior parte dei quali sono ad alto costo.

Circa il $60 \%$ della spesa farmaceutica convenzionata è dovuto alla prescrizione di solo quindici categorie terapeutiche di farmaci, la maggior parte dei quali collocati in Classe A (Tabella 9). In ordine decrescente rispetto al valore assoluto di spesa troviamo al primo posto le cefalosporine e i carbapenemi, al secondo posto i calcioantagonisti selettivi sui vasi, al terzo posto gli antiulcera, al quarto posto gli ACE-inibitori. Questa classifica, fatta eccezione per gli antibiotici, vede nelle prime 15 posizioni le categorie di farmaci indicati per la maggior parte delle patologie croniche che affliggono la popolazione italiana.

Ancora più interessante è analizzare quali categorie terapeutiche abbiano evidenziato un maggiore incremento percentuale della spesa negli ultimi anni. La Tabella 10 riporta le prime 15 categorie terapeutiche che hanno mostrato un maggiore incremento tra il 1997 e il 1998. Gli incrementi percentuali più elevati sono dovuti alla introduzione di nuove categorie di farmaci come gli antagonisti dell' angiotensina II; incrementi consistenti sono anche dovuti al- l'espansione di alcuni settori terapeutici come quello delle dislipidemie e della stessa ipertensione, patologie croniche molto diffuse nella popolazione, ma non ancora saturate come possibilità terapeutiche. Un caso a parte può essere considerato quello dell' eritropoietina, collocata al $15^{\circ}$ posto come incremento di spesa. L'eritropoietina, da farmaco di nicchia riservato ai dializzati, è diventato un farmaco di sempre maggior consumo, utilizzato in molte forme di anemia al posto delle emotrasfusioni. Inoltre, alcune indagini della magistratura hanno evidenziato come almeno una parte dell'incremento del mercato sia dovuto all'uso illecito come sostanza dopante.

L'incremento della spesa farmaceutica convenzionata, del contributo in ticket e del numero di ricette non è uniforme sul territorio nazionale, ma varia sensibilmente nelle varie Regioni, come evidenzia la Tabella 11.

Significativo è anche seguire l'andamento di alcuni indicatori specifici del consumo dei farmaci e della spesa farmaceutica convenzionata, come la spesa annuale procapite (Figura 3), il numero di ricette procapite (Figura 4) e la spesa farmaceutica per ricetta (Figura 5). Rispetto alla media nazionale, le Regioni del Nord tendono ad avere una minore spesa farmaceutica procapite, un minor numero di ricette procapite ed un minor costo per ricetta. Tale

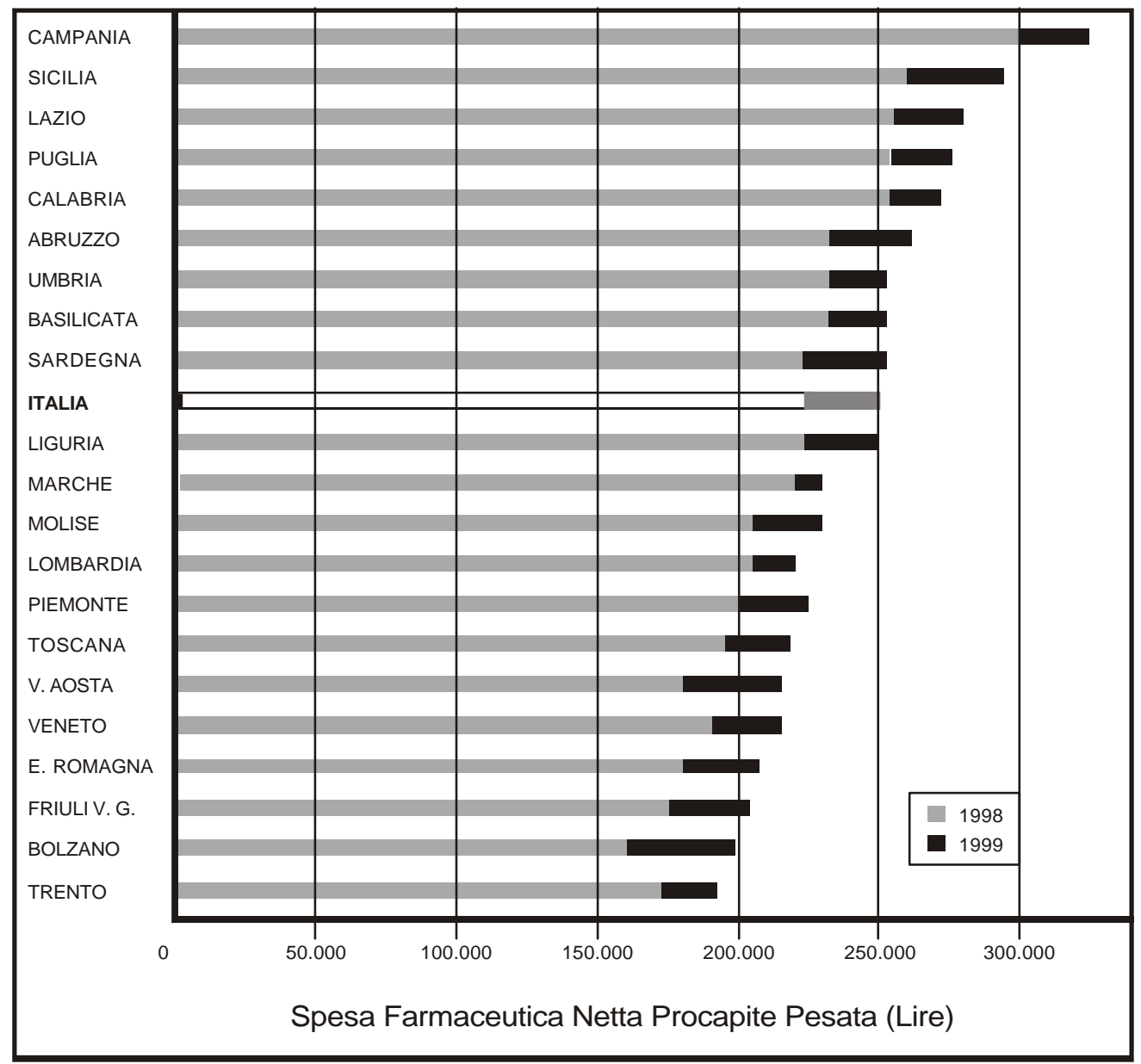

Figura 3

Valori nazionali e regionali della Spesa Farmaceutica Netta Procapite Pesata stimata negli anni 1998-99 (Fonte: Agenzia per $i$ Servizi Sanitari Regionali). 
Figura 4

Valori nazionali e regionali del numero di Ricette

Procapite Pesato, stimato negli anni 1998-99 (Fonte:

Agenzia per i Servizi Sanitari Regionali).
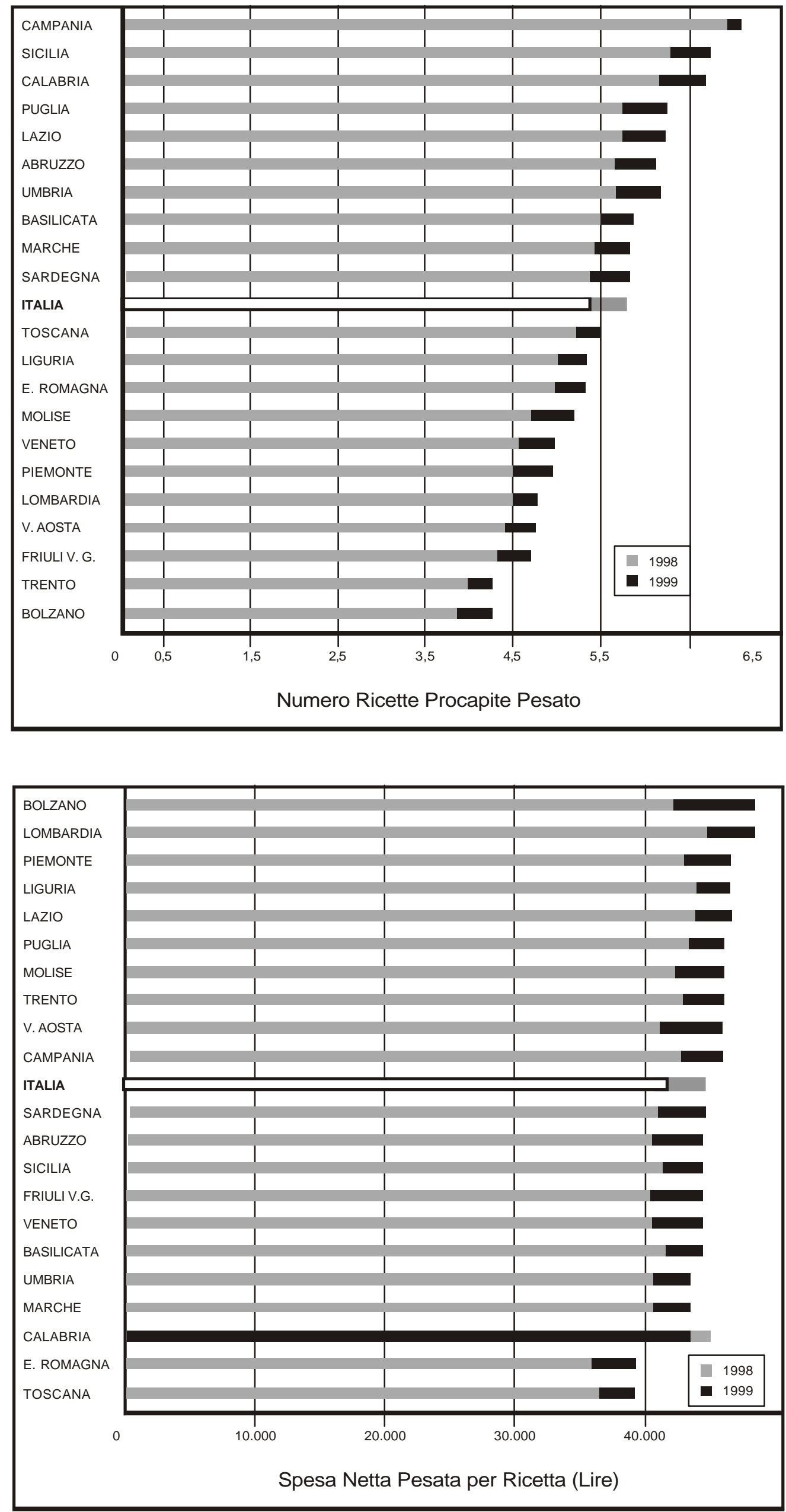

\section{Figura 5}

Valori nazionali e regionali della Spesa Farmaceutica Netta Pesata per ricetta, stimata negli anni 1998-99 (Fonte: Agenzia per $i$ Servizi Sanitari Regionali). 


\begin{tabular}{|c|c|c|c|c|c|c|}
\hline & \multicolumn{3}{|c|}{ Spesa Farmaceutica 1999} & \multicolumn{3}{|c|}{ Spesa Farmaceutica 2000} \\
\hline & $\begin{array}{c}\text { Spesa Netta } \\
\text { (milioni L.) }\end{array}$ & \begin{tabular}{|c||} 
Indicazione \\
F.S.N. 1999 \\
(milioni L.)
\end{tabular} & $\begin{array}{l}\text { Scostamento } \\
\% \text { dal F.S.N. }\end{array}$ & $\begin{array}{c}\text { Previsione } \\
\text { Spesa Netta } \\
\text { (milioni L.) }\end{array}$ & $\begin{array}{c}\text { Indicazione } \\
\text { F.S.N. 2000 } \\
\text { (milioni L.) }\end{array}$ & $\begin{array}{l}\text { Scostamento } \\
\% \text { dal F.S.N. }\end{array}$ \\
\hline V. AOSTA & 26.182 & 26.897 & $-2,7 \%$ & 30.470 & 30.825 & $-1,2 \%$ \\
\hline PIEMONTE & 1.048 .099 & 1.003 .666 & $4,4 \%$ & 1.194 .985 & 1.145 .852 & $4,3 \%$ \\
\hline LIGURIA & 472.578 & 415.988 & $13,6 \%$ & 519.357 & 473.532 & $9,7 \%$ \\
\hline LOMBARDIA & 2.065 .314 & 1.972 .384 & $4,7 \%$ & 2.280 .501 & 2.263 .255 & $0,8 \%$ \\
\hline VENETO & 969.275 & 983.632 & $-1,5 \%$ & 1.098 .638 & 1.125 .979 & $-2,4 \%$ \\
\hline BOLZANO & 87.622 & 94.088 & $-6,9 \%$ & 108.447 & 107.275 & $1,1 \%$ \\
\hline TRENTO & 89.093 & 102.873 & $-13,4 \%$ & 97.557 & 117.812 & $-17,2 \%$ \\
\hline FRIULI V.G. & 260.721 & 283.650 & $-8,1 \%$ & 299.707 & 322.812 & $-7,2 \%$ \\
\hline E. ROMAGNA & 920.520 & 954.668 & $-3,6 \%$ & 1.055 .939 & 1.089 .531 & $-3,1 \%$ \\
\hline MARCHE & 373.166 & 341.905 & $9,1 \%$ & 403.315 & 390.375 & $3,3 \%$ \\
\hline TOSCANA & 855.205 & 849.427 & $0,7 \%$ & 944.258 & 968.901 & $-2,5 \%$ \\
\hline LAZIO & 1.467 .776 & 1.129 .078 & $30,0 \%$ & 1.629 .937 & 1.296 .701 & $25,7 \%$ \\
\hline UMBRIA & 229.254 & 199.612 & $14,8 \%$ & 253.902 & 228.397 & $11,2 \%$ \\
\hline ABRUZZO & 350.410 & 285.993 & $22,5 \%$ & 406.753 & 329.494 & $23,4 \%$ \\
\hline MOLISE & 80.556 & 74.480 & $8,2 \%$ & 93.272 & 85.492 & $9,1 \%$ \\
\hline CAMPANIA & 1.647 .010 & 1.117 .664 & $47,4 \%$ & 1.776 .064 & 1.281 .064 & $38,6 \%$ \\
\hline PUGLIA & 1.058 .510 & 824.558 & $28,4 \%$ & 1.165 .631 & 943.136 & $23,6 \%$ \\
\hline BASILICATA & 149.083 & 128.945 & $15,6 \%$ & 165.952 & 147.544 & $12,5 \%$ \\
\hline CALABRIA & 535.707 & 424.711 & $26,1 \%$ & 582.075 & 485.599 & $19,9 \%$ \\
\hline SICILIA & 1.417 .821 & 1.047 .599 & $35,3 \%$ & 1.609 .741 & 1.201 .061 & $34,0 \%$ \\
\hline SARDEGNA & 391.784 & 338.182 & $15,8 \%$ & 450.865 & 386.361 & $16,7 \%$ \\
\hline ITALIA & 14.496 .356 & 12.600 .000 & $15,1 \%$ & 16.154 .169 & 14.421 .000 & $12,0 \%$ \\
\hline
\end{tabular}

andamento può essere in qualche modo ricollegato ai dati dell'indagine Istat che dimostravano una percezione maggiormente negativa dello stato di salute da parte delle popolazioni del Sud.

Infine, un dato particolarmente significativo è l'analisi, Regione per Regione, dello scostamento della spesa farmaceutica reale da quella prevista dalla programmazione annuale attuata dal Ministero della Sanità (Tabella 12). Le poche Regioni che negli anni 1999 e 2000 hanno realizzato una spesa inferiore alla previsione di spesa programmata si collocano tutte al Nord Italia, mentre al Centro ed al Sud si trovano le Regioni che hanno sforato maggiormente in termini percentuali rispetto al programmato.

L'insieme di questi dati evidenziano la complessità dei fenomeni sociali e culturali che stanoi alla base del consumo di farmaci e più in generale del consumo di risorse sanitarie. In alcune realtà sociali e culturali il contenimento della spesa sembra maggiormente problematica che in altre. L'introduzione diffusa ed acritica di nuove tecnologie (tra queste comprendiamo ovviamente anche i nuovi farmaci, soprattutto quelli biotecnologici) rappresenta un fattore di espansione esplosivo della spesa sanitaria pubblica. Il rimedio migliore per controllare e razionalizzare la spesa sanitaria e quella farmaceutica, in particolare, ritengo debba basarsi su un diffusione sempre maggiore di una corretta cultura medica non disgiunta da una capacità di analisi critica del valore delle innovazioni. Solo così si potranno evitare tagli imposti e sovrastrutture burocratiche che negli ultimi anni hanno appesantito, spesso inutilmente o impropriamente, la professione medica. Solo con maggior cultura medica e maggiore capacità critica il medico potrà essere artefice di uno sviluppo sostenibile della medicina moderna i cui benefici siano equamente resi accessibili a tutti coloro che ne hanno bisogno. 